\title{
RADIATION-DRIVEN ACCELERATION IN PHOTOSPHERES OF NONACCRETING MAGNETIC WHITE DWARFS
}

\author{
Vladimir V. ZhelezNYAKoV AND A. V. SeRBer \\ Institute of Applied Physics, Russian Academy of Science, ul. Ul'yanova 46, 603600 Nizhny Novgorod, Russia \\ Received 1993 February 19; accepted 1993 July 9
}

\begin{abstract}
Radiation transfer in a pure hydrogen, fully ionized, isothermal photosphere of an isolated white dwarf with dipole magnetic field is considered, and the radiation pressure force, both in the continuum and in the cyclotron line, is determined with the line saturation effect taken into account. It is shown that the magnetic field can reduce the critical luminosity for white dwarfs. This leads to the possibility of photospheric plasma ejection driven by the radiation in the cyclotron line and the formation of radiation-driven winds from sufficiently hot isolated magnetic white dwarfs.

It is shown that cyclotron radiation pressure plays a significant role in the force balance of the photospheres of the magnetic white dwarfs GD $229, \mathrm{GrW}+70^{\circ} 8247$, and PG $1031+234$. The strong unidentified depression in the UV spectrum of GD 229 is attributed to cyclotron scattering by the radiation-driven plasma envelope with density $N \gtrsim 10^{8} \mathrm{~cm}^{-3}$.
\end{abstract}

Subject headings: radiative transfer — stars: atmospheres — stars: magnetic fields — white dwarfs

\section{INTRODUCTION}

If the luminosity of a star exceeds the critical, or Eddington, luminosity, $L_{\mathrm{cr}}=1.26 \times 10^{38}\left(M / M_{\odot}\right)$ ergs $\mathrm{s}^{-1}$, or, equivalently, if the surface temperature is greater than the corresponding critical value,

$$
T_{\mathrm{cr}}=6.2 \times 10^{5}\left(\frac{g}{10^{8} \mathrm{~cm} \mathrm{~s}^{-2}}\right)^{1 / 4} \mathrm{~K}
$$

( $g$ is the free-fall acceleration at the star surface), then the effective mass loss due to the Thomson scattering by free electrons may occur. White dwarfs with $g \simeq 10^{8} \mathrm{~cm} \mathrm{~s}^{-2}$ usually have temperatures far below the critical $T_{\mathrm{cr}}^{\mathrm{WD}}=6.2 \times 10^{5} \mathrm{~K}$. However, if a strong magnetic field $B \sim 10^{7}-10^{9} \mathrm{G}$ is present, the situation may change radically.

The magnetic field resonantly enhances the plasma opacity at the fundamental and higher harmonics of gyrofrequency. This results in an increase of the radiation pressure force which can become comparable to or even larger than the force of gravitation (Mitrofanov \& Pavlov 1982; Mitrofanov 1986; Zheleznyakov \& Litvinchuk 1986, 1987). The great radiation force dominated by the resonant cyclotron opacity can drive the ejection and further acceleration of plasma from the photospheres of magnetic white dwarfs. One would expect plasma parameters in the vicinity of these stars to be determined by cyclotron radiation pressure, in the same way as atmospheric parameters of early-type stars are determined by ion resonance lines (Cassinelly 1979).

In what follows we consider radiative transfer and determine the radiation pressure force in the hot photosphere of a magnetic white dwarf, taking into account Thomson scattering and bremsstrahlung emission and absorption for the continuum radiation and also resonant cyclotron scattering and pure emission/absorption inside the cyclotron line at the first harmonic. It is shown that in certain cases the cyclotron-line radia- tion pressure exceeds the gravitational force and causes plasma outflow from the surface of the star.

\section{THE RADIATION PRESSURE FORCE}

The total radiation pressure force per particle in unit volume at rest at a given point in the photosphere of an isolated nonrotating white dwarf with dipole magnetic field,

$$
\boldsymbol{f}=\boldsymbol{f}_{c}+\boldsymbol{f}_{B},
$$

is comprised of the force due to Thomson scattering and bremsstrahlung absorption of radiation at frequencies

$$
\left|\omega-\omega_{B}\right| \geq 3 \sqrt{2} \beta_{T_{1}} \omega_{B}
$$

outside the cyclotron line,

$$
\begin{gathered}
\boldsymbol{f}_{c}=\int_{0}^{\omega-} \boldsymbol{G} d \omega+\int_{\omega_{+}}^{\infty} \boldsymbol{G} d \omega, \\
\boldsymbol{G}=\frac{1}{c}\left(\sigma_{\mathrm{Th}}+\sigma_{b}\right) \sum_{l=1,2} \frac{1+R_{l}}{2} \oint_{4 \pi} I_{\omega l}(\omega, \boldsymbol{n}) \boldsymbol{n} d \Omega,
\end{gathered}
$$

and the force

$$
\begin{aligned}
\boldsymbol{f}_{B}=\frac{2 \pi^{2} e^{2}}{m c^{2}} \frac{2}{\sqrt{\pi}} \int_{0}^{\infty} \Phi(\xi) d \xi \\
\quad \times \int_{-1}^{1} I_{11}(\xi, \boldsymbol{n})\left(1+\cos ^{2} \alpha\right) n d \Omega
\end{aligned}
$$

due to the radiation in the cyclotron line, where

$$
\left|\omega-\omega_{B}\right| \leq 3 \sqrt{2} \beta_{T_{\mid}} \omega_{B}|\cos \alpha|
$$


(Zheleznyakov \& Litvinchuk 1987; Zheleznyakov \& Serber $1991)$. Here $\omega_{ \pm}=\omega_{B}\left(1 \pm 3 \sqrt{2} \beta_{T_{1}}\right) ; \omega_{B}$ is the gyrofrequency; $\sigma_{\mathrm{Th}}$ and $\sigma_{b}$, where

$$
\sigma_{b}=\frac{(4 \pi)^{3} \hbar^{2} e^{6} N}{6 \pi m^{2} c\left(\kappa T_{1}\right)^{3}}\left(\frac{m}{2 \pi \kappa T_{1}}\right)^{1 / 2} u^{-3}\left(1-e^{-u}\right) F,
$$

are the Thomson and bremsstrahlung cross sections in an isotropic plasma, respectively (Bekefi 1966);

$$
F= \begin{cases}\ln \left[(2 / \gamma)^{5 / 2} \beta_{T_{1}} / u\right] & \text { if } u \leq\left(\hbar c / e^{2}\right) \beta_{T_{1}} \\ 1 & \text { otherwise }\end{cases}
$$

is the classical Gaunt factor valid for $T_{1} \leq 8.9 \times 10^{5} \mathrm{~K} ; T_{\|}=$ $T$ is the longitudinal (relative to the magnetic field) plasma temperature; $\beta_{T_{1}}=\left(\kappa T_{\|} / m c^{2}\right)^{1 / 2} ; u=\hbar \omega / \kappa T_{\|} ; \gamma=1.781$ is the Euler constant; the factors $R_{L}=\omega^{2}\left(\omega \mp \omega_{B}\right)^{-2}$ approximately describe the effect of the magnetic field on the bremsstrahlung and Thomson cross sections (the minus sign corresponds to the extraordinary mode $l=1$ and the plus sign to the ordinary mode $l=2) ; I_{\omega l}(\omega, n)$ is the spectral intensity of radiation in mode $l ; \Omega$ is the solid angle; $n$ is the unit vector along a ray; $\alpha$ is the angle between $\boldsymbol{n}$ and $\boldsymbol{B}$;

$$
I_{11}(\xi, \mu)=\int_{0}^{\infty} I_{\omega 1}(\omega, \mu) \delta\left[\omega\left(1-\sqrt{2} \beta_{T_{1}} \xi \mu\right)-\omega_{B}\right] d \omega
$$

is the intensity of extraordinary radiation at the first cyclotron harmonic interacting with electrons which have longi-

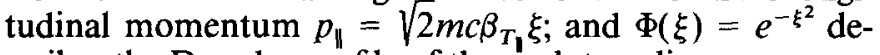
scribes the Doppler profile of the cyclotron line.

Note that equation (3) does not take into account the angular dependence of the Thomson cross section in the magnetic field or the shift of the photon frequency due to Doppler and Compton effects. Note also that for typical white dwarf parameters the following condition is well satisfied:

$$
\frac{1}{45 \pi} \frac{e^{2}}{\hbar c}\left(\frac{B}{B_{c}}\right)^{2} \frac{\beta_{T_{1}} \omega_{B}^{2}}{\omega_{p}^{2}} \simeq 10^{23} T^{1 / 2} N^{-1}\left(\frac{B}{B_{c}}\right)^{4} \ll 1,
$$

where $\omega_{p}$ is the plasma frequency and $B_{c}=m^{2} c^{3} / \hbar e \simeq$ $4.14 \times 10^{13} \mathrm{G}$, so the modes polarization is determined by the rarefied plasma and not by the vacuum birefringence in a strong magnetic field (Zheleznyakov 1984). In this case the ordinary mode opacity is much less than the extraordinary one:

$$
\chi_{12} / \chi_{11} \sim \beta_{T_{1}}^{2} \ll 1 ;
$$

the ordinary radiation weakly interacts with plasma, and so its influence on the force given by equation (4) has been neglected.

The radiation intensities in equations ( 3 ) and (4) should be determined by solving the corresponding equations of radiation transfer. In doing this, we shall assume that radiation does not enter the photosphere from the outside, while deep in the photosphere the radiation intensity remains finite.

For the sake of simplicity we consider here an isothermal, fully ionized, pure hydrogen photosphere in hydrostatic equilibrium. Then, over distances much shorter than the stellar radius $R_{*} \sim 10^{9} \mathrm{~cm}$, the plasma density decreases with height according to the barometric law

$$
N \propto \exp (-z / H),
$$

where $H=2 \kappa T / m_{p} g$ is the photospheric scale height, $m_{p}$ is the proton mass, $\kappa$ is Boltzmann's constant, and the $z$-axis points away from the star. For white dwarfs $T<10^{6} \mathrm{~K}$ and $g \simeq 10^{8}$ $\mathrm{cm} \mathrm{s}^{-2}$. Under these conditions $H \ll \min \left(R_{*}, l_{B}\right)$ where $l_{B} \simeq$ $\sqrt{2} \beta_{T_{1}} L_{B}$ is the thickness of the gyroresonant layer (Zheleznyakov 1984; Zheleznyakov \& Litvinchuk 1986), $L_{B}$ is the characteristic scale of the magnetic field $\left(L_{B} \sim R_{*} / 3\right.$ for a dipole field). The magnetic field can therefore be assumed uniform, the gravitational force constant, and the plasma photosphere a plane-parallel medium. Note that for any point of the star surface except the poles the magnetic field direction differs from radial, the angle between $B$ and the $z$-axis being equal to

$$
\delta=\arccos \left[\frac{2 \cos \Theta}{\left(1+3 \cos ^{2} \theta\right)^{1 / 2}}\right],
$$

where $\Theta$ is the angle between $\hat{z}$ and the magnetic axis of the star ( $\hat{z}$ is the unit vector along the $z$-axis). Transfer of radiation in planar photospheres of magnetic degenerate stars for the case $\delta=0$ has been studied in detail by Zheleznyakov (1981) and Zheleznyakov \& Serber (1991). Here we use previous results to investigate the general case $\delta \neq 0$.

The continuum radiation at frequencies given by equation (2) is affected by bremsstrahlung and Thomson scattering. Zheleznyakov \& Serber (1991) have shown that for white dwarf parameters the bremsstrahlung cross section is larger than the Thomson one, $\sigma_{b}>\sigma_{\mathrm{Th}}$, so the continuum radiation intensity is dominated by bremsstrahlung, while the scattering can be neglected. In other words, the continuum radiation of the magnetic white dwarf photosphere is formed under LTE conditions. The corresponding equations of transfer for modes 1 and 2 has the form

$$
\mu \frac{\partial I_{\omega l}\left(\tau_{b}, \omega, \mu\right)}{\partial \tau_{b}}=\frac{1+R_{l}}{2}\left[-I_{\omega l}\left(\tau_{b}, \omega, \mu\right)+B_{\omega}(\omega)\right] .
$$

In this equation

$$
B_{\omega}(\omega)=\frac{\hbar \omega^{3}}{8 \pi^{3} c^{2}}\left[\exp \left(\frac{\hbar \omega}{\kappa T}\right)-1\right]^{-1}
$$

is the intensity of blackbody radiation at temperature $T$ in one mode, $\tau_{b}=\sigma_{b} N H / 2$ is the bremsstrahlung optical depth, $\mu=$ $\cos \theta$, and $\theta$ is the angle between $n$ and $\hat{z}$.

For an isothermal medium the solution of equation (7) is easy to find (Zheleznyakov \& Serber 1991), and the contin- 
uum radiation pressure force in this case is determined by equation (3), in which

$$
\begin{aligned}
\boldsymbol{G}=\hat{z} \cdot \frac{\pi}{c}\left(\sigma_{\mathrm{Th}}+\sigma_{b}\right) \frac{B_{\omega}(\omega)}{2} & \\
& \times \sum_{l=1,2}\left(1+R_{l}\right) \exp \left[-\frac{\tau_{b}\left(1+R_{l}\right)}{2}\right] .
\end{aligned}
$$

Note that $f_{c}$ is directed radially outward from the star.

Transfer of extraordinary radiation at the cyclotron fundamental is described by the equation

$$
\begin{aligned}
|\cos \alpha| \mu & \frac{d I_{11}(\xi, \mu, \phi)}{d \tau} \\
& =\frac{1+\cos ^{2} \alpha}{4} \Phi(\xi)\left[I_{11}(\tau, \xi, \mu, \phi)-S_{1}(\tau, \xi)\right],
\end{aligned}
$$

where

$$
\begin{aligned}
S_{1}(\tau, \xi)=\frac{1}{1+\epsilon} \int_{-1}^{1} d \mu & \int_{0}^{2 \pi} d \phi \frac{3}{16 \pi}\left(1+\cos ^{2} \alpha\right) \\
& \times I_{11}(\tau, \xi, \mu, \phi)+\frac{\epsilon}{1+\epsilon} B_{\omega}\left(\omega_{B}\right)
\end{aligned}
$$

is the source function,

$$
\omega_{B}=\omega_{B_{p}} \frac{\left(1+3 \cos ^{2} \Theta\right)^{1 / 2}}{2}
$$

is the local gyrofrequency at the star surface, $\omega_{B_{p}}$ is the gyrofrequency corresponding to the polar field $B_{p}$,

$$
\cos \alpha=\mu \cos \delta+\left(1-\mu^{2}\right)^{1 / 2} \sin \delta \cos \phi,
$$

$\phi$ is the angle between $\boldsymbol{n}$ and the $(\hat{\boldsymbol{z}}, \boldsymbol{B})$-plane, and

$$
\tau=\frac{4 \sqrt{2} \pi^{3 / 2} e^{2}}{m c \beta_{T_{1}} \omega_{B}} N H
$$

is the line-center optical depth measured from the top of the photosphere. The parameter

$$
\epsilon \simeq 1.25 \times 10^{9} N K B^{-2} T_{\|}^{-3 / 2}=M_{B} \tau
$$

where

$$
M_{B}=6.4 \times 10^{-4}\left(\frac{g}{10^{8} \mathrm{~cm} \mathrm{~s}^{-2}}\right)\left(\frac{B}{10^{8} \mathrm{G}}\right)^{-1}\left(\frac{T}{10^{4} \mathrm{~K}}\right)^{-2}
$$

is the ratio of the spontaneous cyclotron transition time to the time between collisions ( $K \sim 10$ is the Coulomb logarithm). Terms proportional to $\epsilon /(1+\epsilon)$ describe pure absorption due to radiative excitation of Landau levels with subsequent collisional de-excitation, as well as pure emission due to collisional excitation of Landau levels with subsequent radiative de-excitation. Under the white dwarf conditions these processes influ- ence the cyclotron line radiation much more strongly than bremsstrahlung emission/absorption (Zheleznyakov \& Litvinchuk 1984). Since $\epsilon \ll 1$ in white dwarf photospheres, the scattering dominates over the absorption, and the extraordinary radiation at the cyclotron fundamental is formed in the non-LTE conditions. It is clear that in this model of the photosphere the cyclotron line in absorption is formed in the extraordinary radiation at the cyclotron fundamental in a manner similar to the formation of Fraunhofer lines in the optical spectra of the ordinary stars. The feature of the spectra of a magnetic white dwarf is that the line appears as a result of the electron transitions up and down between the Landau levels in a magnetic field.

Let us find the radiation intensity and the force (eq. [4]) in the Schwarzschild-Schuster approximation (see, e.g., Mihalas 1978 ) when equation ( 8 ) is reduced to the system

$$
\pm \frac{d I_{11}^{(1),(2)}}{d \tau}=\Phi(\xi) \mathcal{f}(\delta)\left(I_{11}^{(1),(2)}-S_{1}\right)
$$

for the angle-averaged intensities

$$
\begin{aligned}
& I_{11}^{(1)}=\int_{0}^{1} d \mu \int_{0}^{\pi} d \phi I_{11}(\tau, \xi, \mu, \phi), \\
& I_{11}^{(2)}=\int_{-1}^{0} d \mu \int_{0}^{\pi} d \phi I_{11}(\tau, \xi, \mu, \phi) .
\end{aligned}
$$

In these equations

$$
S_{1}=\frac{1}{1+\epsilon} \frac{I_{11}^{(1)}+I_{11}^{(2)}}{2}+\frac{\epsilon}{1+\epsilon} B_{\omega}\left(\omega_{B}\right),
$$

and the factor (Litvinchuk 1987)

$$
\mathscr{f}(\delta)=\frac{1}{2}\left[\frac{\sin \delta}{\pi}+\frac{\cos \delta}{2}\left(1-\frac{2 \delta}{\pi}\right)\right]^{-1}
$$

ranges from 1 at the magnetic poles to $\pi / 2$ at the equator. It describes the influence of the magnetic field direction on the optical depth of the photosphere. Note that the system (11) differs only by the factor $f(\delta)$ from the similar system for the case $\delta=0$, which has been treated in detail by Zheleznyakov (1981) and Zheleznyakov \& Serber (1991). Therefore, the analytical solution of the system (11) for $\epsilon \ll 1$ can be written immediately using the known result for $\delta=0$. Substituting $I_{11}(\tau, \xi, \mu, \phi) \simeq I_{11}^{(1)}(\tau, \xi)$ for $\cos \theta>0$ and $I_{11}(\tau, \xi, \mu, \phi) \simeq$ $I_{\text {II }}^{(2)}(\tau, \xi)$ for $\cos \theta<0$, we obtain the force (eq. [4]) in the Schwarzschild-Schuster approximation:

$$
\begin{aligned}
& f_{B}=\left(\hat{z} \frac{5+\cos ^{2} \delta}{6}+\hat{\theta} \frac{\sin \delta \cos \delta}{3}\right) f_{B}, \\
& f_{B}(\tau)=\frac{3 \pi^{3} e^{2}}{m c^{2}}\left(\frac{M_{B}}{f}\right)^{1 / 3} B_{\omega}\left(\omega_{B}\right) \frac{2}{\sqrt{\pi}} \\
& \times \int_{0}^{\infty} \frac{-2 \mathrm{Ai}^{\prime}\left((\Phi /)^{2 / 3} M_{B}^{1 / 3} \tau\right)}{\operatorname{Ai}(0) \Phi^{1 / 3}-\mathrm{Ai}^{\prime}(0)\left(M_{B} / f\right)^{1 / 3}} \Phi d \xi .
\end{aligned}
$$


where $\operatorname{Ai}(x)$ and $\operatorname{Ai}^{\prime}(x)$ are the Airy function and its derivative, respectively. The azimuthal component of $f_{B}$ is absent because of the axial symmetry of the star. The longitudinal and transverse components of the radiation force (eq. [1]) are given by

$$
\begin{aligned}
& f_{\|}=\left(f_{c}+f_{B} \frac{7-\cos ^{2} \delta}{6}\right) \cos \delta, \\
& f_{\perp}=\left(f_{c}+f_{B} \frac{5-\cos ^{2} \delta}{6}\right) \sin \delta .
\end{aligned}
$$

The force in equation (1) is maximal at the top of the photosphere:

$$
\begin{aligned}
& f_{B}^{\max } \simeq \frac{3 \pi^{3} e^{2}}{m c^{2}}\left(\frac{M_{B}}{f}\right)^{1 / 3} B_{\omega}\left(\omega_{B}\right) \frac{-\mathrm{Ai}^{\prime}(0) \sqrt{6}}{\mathrm{Ai}(0)}, \\
& f_{c}^{\max } \simeq \frac{\sigma_{\mathrm{Th}}}{c}\left[\sigma T^{4}+\pi B_{\omega}\left(\omega_{B}\right) \frac{\omega_{B}}{3 \sqrt{2} \beta_{T_{I}}}\right]
\end{aligned}
$$

(here $\sigma$ is the Stefan-Boltzmann constant) and rapidly decreases with increasing $N$.

\section{THE CRITICAL LUMINOSITY OF MAGNETIC WHITE DWARFS}

The photospheric plasma can move freely along the magnetic field lines under the action of the radiation pressure force and the force of gravity, while the transverse motion in the strong magnetic field of the white dwarf is suppressed, and the action of the transverse components of radiation and gravity forces results only in the drift current in the photosphere. Radiation pressure force does not disrupt the photospheric equilibrium if

$$
\Gamma=\frac{f_{1}^{\max }}{m_{p} g \cos \delta}<1 .
$$

The solid curves in the $B-T$ plane in Figure 1 correspond to the conditions $\Gamma=1,10,100$ for $g=10^{8} \mathrm{~cm} \mathrm{~s}^{-2}$ and $\delta=0$ (the magnetic pole), while the dashed curves correspond to $\delta=\pi / 2$ (the equator). To the right of the curves $\Gamma=1$ the hydrostatic equilibrium of the photosphere is impossible. The vertical dashed line shows the value of $T_{\mathrm{cr}}^{\mathrm{WD}}$. From this figure it is clear that the magnetic fields in the interval $10^{8}-10^{10} \mathrm{G}$ significantly reduce the maximum value of the temperature for which the photosphere can stand against the radiation pressure.

The strong magnetic field can cause the wind from the white dwarf photospheres driven by radiation in the cyclotron line. Therefore, isolated magnetic white dwarfs with $B \simeq 10^{8}-10^{10}$ $G$ should exhibit enhanced mass loss compared with the nonmagnetic ones with the same parameters. Another possible class of objects with photospheric outflow could be young hot white dwarfs ( $T \gtrsim 10^{5} \mathrm{~K}$ ) born during the evolution of planetary nebula nuclei.

Plasma ejected from the photosphere along the magnetic field lines forms an extended envelope and/or equatorial disk in a white dwarf magnetosphere. Such an object consisting of a hot magnetic star and the disklike plasma envelope supported

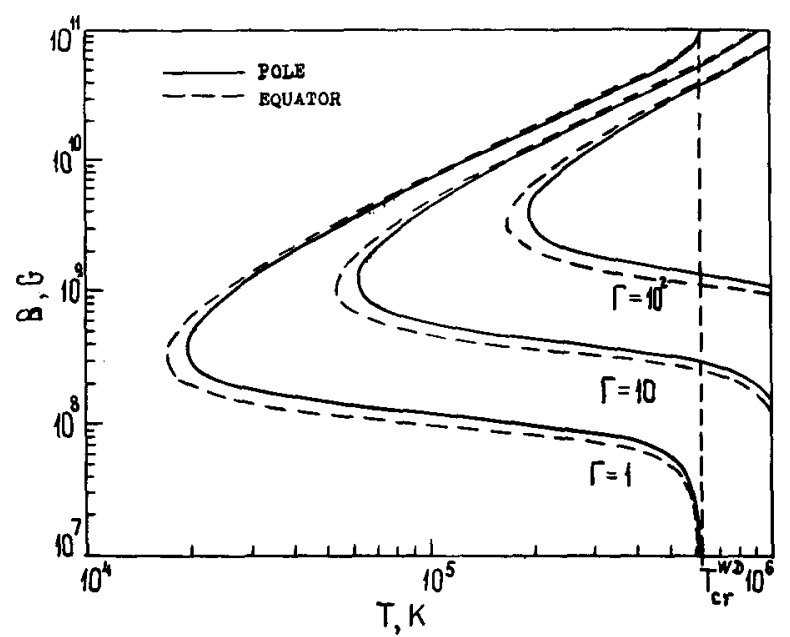

FIG. 1.-Plot of magnetic field vs. temperature for $\Gamma=1,10$, and 100 and for $\delta=0$ and $\delta=\pi / 2$.

by the radiation pressure have been called radiation-driven diskon (Bespalov \& Zheleznyakov 1990; Bespalov, Serber, \& Zheleznyakov 1990). The radial diffusion of plasma is provided by flute instability. The envelope may influence the radiation of the star leading to the formation of the features in the apparent radiation spectrum of the whole object.

\section{DISCUSSION}

We have inspected the list of magnetic white dwarfs collected in Schmidt (1989) and have found three stars for which the cyclotron radiation pressure is the large fraction of, or is even greater than, the gravity force.

1. GrW $+70^{\circ} 8247$ has $B_{p} \simeq 3.2 \times 10^{8} \mathrm{G}$ and the temperature $T \simeq 1.4 \times 10^{4} \mathrm{~K}$, so $\Gamma(\delta=0) \simeq 0.5 \simeq \Gamma(\delta=\pi / 2)$.

2. For PG $1031+234$, with $B_{p} \simeq 5 \times 10^{8} \mathrm{G}$ and $T \simeq 1.5 \times$ $10^{4} \mathrm{~K}$, the factor $\Gamma(\delta=0) \simeq 0.4$ and $\Gamma(\delta=\pi / 2) \simeq 0.8$.

3 . The third star, GD 229 , has a very complicated spectrum with a number of absorption lines in the optical band and immense depression covering the interval $\sim 2000-3000 \AA$ (Green \& Liebert 1981; Schmidt, Latter, \& Foltz 1990). The optical energy distribution matches best a temperature of $20,000 \mathrm{~K}$, and the UV spectrum yields a smaller value near $16,000 \mathrm{~K}$. So we have used these values as upper and lower estimates of the star temperature. We believe that the most viable explanation for the UV feature is cyclotron scattering at the first harmonic by the gyroresonant layer with the optical depth (Zheleznyakov 1984; Zheleznyakov \& Litivinchuk 1986)

$$
\tau_{g}=\frac{2 \pi^{2} e^{2} N L_{B}}{m c \omega_{B}}\left(1+\cos ^{2} \alpha\right) \gtrsim 1
$$

situated in the radiation-driven plasma envelope at a distance $r_{g}$ from the star center. If the blue wing of the UV depression corresponds to the fields near the polar one, then $B_{p} \simeq 5.3 \times$ $10^{8} \mathrm{G}$. Taking for the estimate $r_{g} \sim R_{*} \sim 10^{9} \mathrm{~cm}$, we find that 
condition (14) is met if the plasma density $N \geqslant 10^{\triangleleft} \mathrm{cm}^{-3}$. We also find that $\Gamma(\delta=0) \simeq 0.45-0.93$ and $\Gamma(\delta=\pi / 2) \simeq$ $0.88-1.26$.

Note that the curves in Figure 1 have been drawn for the cyclotron radiation force in the Schwarzschild-Schuster approximation, and the exact value of $f_{B}^{\max }$ may differ from expression (13). Note also that the photospheric outflow may occur even in the region $\Gamma \lesssim 1$. Indeed, a small longitudinal velocity perturbation of a plasma element in the upper photosphere leads to the Doppler shift of the cyclotron line profile into the neighboring continuum and, consequently, to the sharp increase of the radiation pressure force. This enhanced force accelerates a plasma element with the velocity directed outside the star. As a result, an instability may develop which will disrupt the equilibrium of the photosphere with the parameters in the $\Gamma \lesssim 1$ region. The similar instability for the boundary layer of the neutron star photosphere have been considered by Mitrofanov (1986).

Therefore, we can conclude that the photospheres of GD $229, \mathrm{GrW}+70^{\circ} 8247$, and PG $1031+234$ are at the stability threshold against cyclotron radiation pressure.

The detailed analysis of the structure and dynamics of the radiation-driven envelopes around magnetic white dwarfs will be the subject of subsequent publications.

\section{REFERENCES}

Bekefı G. 1966, Radiation Processes in Plasmas (New York: Wiley) Bespalov, P. A., Serber, A. V., \& Zheleznyakov, V. V. 1990, in Proc. Joint Varenna-Abastumani-Nagoya-Potsdam ESA Int. School and Workshop on Plasma Astrophysics (ESA SP-311; Noordwijk: ESA), 309

Bespalov, P. A., \& Zheleznyakov V. V. 1990, Soviet Astron. Lett., 16, 442 Cassinelly, J. P. 1979, ARA\&A, 17, 275

Green, R. F., \& Liebert, J. 1981, PASP, 93, 105

Litvinchuk, A. A. 1987, Ph.D. thesis, Inst. Applied Physics, Russian Academy of Science

Mihalas, D. 1978, Stellar Atmospheres (San Francisco: Freeman)

Mitrofanov, I. G. 1986, Soviet Astron., 30, 571
Mitrofanov, I. G., \& Pavlov, G. G. 1982, MNRAS, 200, 1033

Schmidt, G. D. 1989, in IAU Colloq. 114, White Dwarfs, ed. G. Wegner (Berlin: Springer-Verlag), 305

Schmidt, G. D., Latter, W. B., \& Foltz, C. B. 1990, ApJ, 350, 758

Zheleznyakov, V. V. 1981, Ap\&SS, 77, 279

. 1984, Soviet Sci. Rev., E, Astrophys. Space Phys.., 3, 157

Zheleznyakov, V. V., \& Litvinchuk, A. A. 1984, Ap\&SS, 105, 73

. 1986, in Proc. Joint Varenna-Abastumani Int. School and Work-

shop on Plasma Astrophysics (ESA SP-311: Noordwijk: ESA), 375

. 1987, Soviet Astron., 31, 159

Zheleznyakov, V. V., \& Serber, A. V. 1991, Soviet Astron. Lett., 17, 179 